\title{
Securing jammed network using reliability behavior value through neuro-fuzzy analysis
}

\author{
S RAJA RATNA ${ }^{1, *}$ and R RAVI ${ }^{2}$ \\ ${ }^{1}$ Department of Information and Communication Engineering, Anna University, \\ Chennai 600 025, India \\ ${ }^{2}$ Department of Computer Science and Engineering, Francis Xavier Engineering \\ College, Tirunelveli 600 025, India \\ e-mail: gracelinrr@yahoo.com; csehod@francisxavier.ac.in
}

MS received 11 November 2014; revised 30 December 2014; accepted 6 February 2015

\begin{abstract}
Wireless multi-hop networks are often exposed to serious physical layer jamming attack. In this attack, the jammer node corrupts the packet by injecting high level of noise and keeps the channel busy and thus blocks the legitimate communication. If multiple jammers collude together, this attack will become very severe. To prevent this attack, a simple yet effective Reliability Behavior Neuro-Fuzzy system has been proposed and it operates in three modules. In module one, each route node obtains its behavior value from the route path and neighboring paths using direct and indirect behavior observations. In module two, based on the behavior value, three factor identification methods have been presented to identify the reliability value of nodes. In module three, using the reliability value the route nodes are level positioned and classified into groups by a neuro-fuzzy classifier. By simulation studies, it is observed that the proposed scheme significantly not only identifies misbehaving nodes with higher detection rate and lower false positive and but also achieves higher network throughput and lower jamming throughput.
\end{abstract}

Keywords. Attacker; behavior; jamming; positioning; reliability; suspicious.

\section{Introduction}

Owing to the transparency of wireless medium together with emerging wireless technologies, the increasing users become vulnerable to physical layer jamming attacks (Amariucai 2009; Shiu et al 2011). The attacker blocks the legitimate communications by effectively and efficiently destroying the transmitted packet by injecting short jamming burst (Noubir \& Lin 2003). Traditionally, threats are addressed using cryptographic methods; however, these attacks cannot be addressed through conventional security mechanisms. Therefore, an effective countermeasure has to be taken to prevent this type of attack.

*For correspondence 
The most important requirement for data transmission is reliability. Each node transmits to its neighboring node by depending on its reliability trust. Reliability trust is the reliability on which a trusting party expects that trusted party to perform a given action correctly on which its welfare depends (Josang et al 2007). An effective countermeasure to jamming attack is to obtain the reliability value for all the nodes in the transmission path and to forward the packet to a node with good value. To subdue this attack, Reliability Behavior Neuro-Fuzzy system is proposed. The objective of this work is to prevent jamming attack by identifying and eliminating the node that performs malicious activity in the transmission path. Prevention of jamming is not feasible without detection.

In the proposed system, a scheme has been presented that allows each node to determine its reliability by calculating the behavior value using both direct and indirect observations. Three factor identification methods have been presented to identify the reliability value of the node, and the nodes are categorized into groups based on their reliability value. Malicious node is then identified and eliminated from the path.

The paper proceeds as follows. Section 2 describes related research work. Section 3 explains the problem statement and assumptions of the proposed work. Section 4 explains the behavior pair establishment, while Section 5 describes the reliability factor identification methods and Section 6 describes the node positioning classification (NPC). Section 7 presents the performance evaluation of Reliability Behavior Neuro-Fuzzy system and summarizes the result. Finally, Section 8 concludes the paper.

\section{Related research work}

The focus of this work is to detect and to prevent the reactive jammer. Many prior works are available that aid in preventing jamming attack like spread spectrum (Chiang \& Hu 2011), frequency hopping (Li et al 2012; Pelechrinis et al 2010), multi-path routing (Furdek \& Kapov 2013; Mustafa et al 2012), packet hiding (Proano \& Lazos 2012) and protocol-based technique (Sarker \& Mouftah 2012). But these works do not help in performing both direct and indirect observations as well as do not calculate the reliability of nodes in the communication path, which is different from the problem of finding the network activities that are associated with an attack.

Ratna et al (2014) in the previous work have proposed Neuro Fuzzy Detachment Scheme to prevent reactive jamming attack that targets the physical layer of the wireless network. In this scheme, the malicious node is identified based on the trust values obtained from its route path maintained at the previous history log files. The limitation of this scheme is that there is possibility for an innocent node to be framed as malicious because the trust value is decided by the route path and not by the neighboring paths. The proposed work differs from this scheme since it receives the trust values both from its route path and its neighboring paths by direct and indirect behavior observation respectively. It also provides better network performance at higher detection rate and lower false positive probability than the previous work.

Nguyen et al (2011) have proposed a framework for identifying the reactive jamming nodes in wireless LAN. The framework relies on 'alibi' in which the detector collects good proofs of nodes to infer the compromised nodes. It deals with both non-colluding and colluding attackers in dense networks. The main drawback of this approach is that the detection probability of colluding attacker is worse when compared to the non-colluding attackers and the nodes cannot send all the proofs to the central detector due to the low throughput of the timing channel.

Spuhler et al (2014) have proposed a method to detect reactive jammers that target the physical layer header of direct sequence spread spectrum (DSSS) packets in wireless communication 
systems. The key idea is to extract information from the jamming-free symbols of the DSSS synchronizer to detect the jammed packets. However, they do not propose any technique to detect jamming if a single extra bit which is added to the packet.

Richa et al (2013) proposed medium access protocol (MAC) protocol ANTIJAM to mitigate reactive jamming attacks that can determine whether the channel is currently idle or busy. It requires no knowledge about the number of participants in the network. This scheme will be efficient only if the number of non-jammed time steps is high, if not, throughput gets decreased. The proposed work differs from this approach since it operates at the physical layer rather than the MAC. Unlike the proposed work, these techniques are not able to detect jammers that affect only a few bits per packet.

Adnane et al (2013) have presented a trust-based solution for securing OLSR protocol and to detect misbehaving nodes. To detect misbehaving nodes, trust-based reasoning has been developed by correlating information provided in the OLSR messages received from the network. The integration of this reasoning allows each node to check the consistency of the behavior of other nodes and validate the trust relationships. Limitation of this approach is that the detection rate becomes lower because the simulation time is not enough to detect the attack.

Finally, Bao et al (2012) proposed a cluster-based hierarchical trust management protocol for wireless sensor networks to deal with malicious nodes. The author demonstrated the feasibility of dynamic hierarchical trust management using trust-based IDS applications. But implementing this complex scheme at every member in a cluster is very complicated.

The salient features of the proposed scheme go beyond the prior research works in terms of the following aspects: (i) a node receive its behavior value both through direct and indirect observations rather than by direct method as in prior works. (ii) Prior works receive the feedback values and just aggregate them to produce the trust factor. (iii) There is very less possibility for an innocent node being framed as bad because the behavior value is decided by both the route path and the neighboring paths. (iv) To enhance the network performance, the proposed scheme calculates reliability using three metrics and three factor identification methods. (v) This system is capable of improving the system efficiency by lowering the jammer throughput while increasing the network throughput.

\section{Network topology model and assumptions}

\subsection{Problem statement}

Consider the nodes $u$ and $v$ which communicate through wireless medium with $u$ being the source and $v$ the sink. A jammer is present in the communication path between $u$ and $v$ intensely listening to all network activities. When $u$ transmits a packet to $v$, the jammer corrupts the packet by injecting large amount of error bits into it. The objective of this work is to identify and to eliminate the malicious node in the transmission path between $u$ and $v$, thereby allowing the packet to reach the receiver side safely.

\subsection{Network and attacker model}

A wireless network consisting of $N$ cooperative nodes connected through wireless links communicating over a single frequency is considered for analysis. If nodes are within the communication range of each other, they communicate directly or communicate via multi-hop. A scenario has been created where the nodes continuously send packet on the wireless channel. 


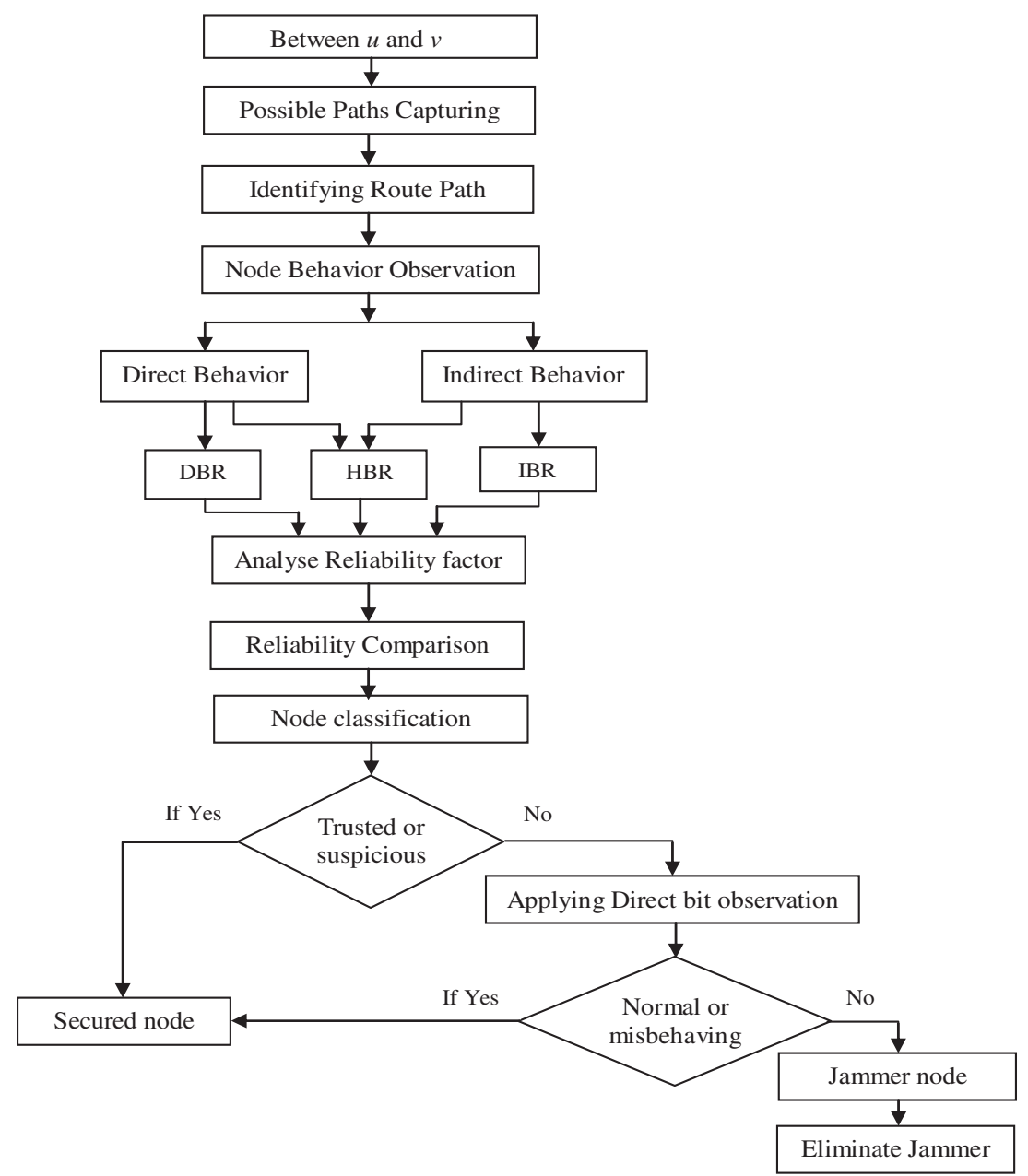

Figure 1. Framework of reliability behavior neuro-fuzzy system.

A jammer is placed in the multi-hop path between the source and the sink and is capable of attacking any part of the packet. Being superior to normal nodes, the jammer node can transmit and receive simultaneously in full duplex mode. In this paper, the attacker is considered to be reactive knowing all network secrets.

\subsection{Overview of Reliability Behavior Neuro-Fuzzy system}

The proposed Reliability Behavior Neuro-Fuzzy system is responsible for identifying the malicious node using node's reliability value. It is the integration of three modules, the Behavior Pair Establishment module, the Reliability Factor Identification module and the Node Positioning Classification module. The framework of the proposed system is shown in figure 1.

- In Behavior Pair Establishment module, the behavior value is calculated for all the route nodes in the route path using direct and indirect observations. 
- In Reliability Factor Identification module, three factor identification methods have been proposed for identifying the reliability of a route node.

- In Node Positioning Classification module, route nodes are classified into groups and the malicious node is identified and eliminated.

\section{Behavior pair establishment}

\subsection{System initialization}

The purpose of system initialization is to capture all possible paths between $u$ and $v$ with a jammer in between them. For each captured path, the throughput, the packet delivery ratio and the delay are evaluated by the number of successful and unsuccessful interactions for a particular time interval. The path with lowest throughput, lowest packet delivery ratio and highest delay is considered to be a malicious path. To specifically identify the jammer node, the reliability value is calculated for all the nodes in the malicious path and the node with the lowest value is considered as a jammer node. Reliability is obtained by first calculating the node's behavior value from the malicious path and from its neighboring paths. It is based on two observations, the Direct Behavior Observation and the Indirect Behavior Observation.

\subsection{Direct behavior observation evaluation}

In Direct Behavior Observation, the malicious path is the route path $r p$ and its nodes are identified as route nodes $r n$. The route path $r p$ with $k$ forwarding nodes is denoted as $r p=$ $\left\{u, r n_{i}, \ldots, r n_{k}, v\right\}$. The source $u$ and the sink $v$ are denoted as $r n_{i-1}$ and $r n_{k+1}$ respectively. In Direct Behavior Observation, each route node obtains the behavior value from its route path and each route node's behavior value $D b_{v}$ is calculated directly from its one hop previous route node. The two $D b_{v}$ 's are $\partial p_{(r i-1, r i)}$ and $l \partial_{(r i-1, r i)}$, where $\partial p_{(r i-1, r i)}$ and $l \partial_{(r i-1, r i)}$ are the probability of trust and link delay respectively. The $\partial p_{(r i-1, r i)}$ and $l \partial_{(r i-1, r i)}$ values are calculated based on the number of successful and unsuccessful interactions between any two consecutive nodes, for example $r n_{i-1}$ and $r n_{i}$.

The link between the source and the first forwarder is $l\left(r n_{i-1}, r n_{i}\right)$. Likewise, the link between $r n_{i-1}$ and $r n_{k+1}$ is given as $\left\{l\left(r n_{i-1}, r n_{i}\right), l\left(r n_{i}, r n_{i+1}\right), \ldots, l\left(r n_{k-1}, r n_{k}\right), l\left(r n_{k}, r n_{k+1}\right)\right\}$. The link delay is the delay that occurs while transferring the packet across the link $l\left(r n_{i-1}, r n_{i}\right)$. The probability of trust given in (1) is calculated based on the number of packets tampered $p_{t(i-1, i)}$, the number of packets dropped $p_{d(i-1, i)}$ and the total number of packets forwarded $T_{f(i-1, i)}$ from $r n_{i-1}$ torn $i$ as in (Shila et al 2010).

$$
\partial p_{(r i-1, r i)}=1-\left(\frac{p_{t(i-1, i)}+p_{d(i-1, i)}}{T_{f(i-1, i)}}\right) .
$$

The calculated two $D b_{v}$ 's are aggregated, the first $\partial p_{(r i-1, r i)}$ and the second $l \partial_{(r i-1, r i)}$. The aggregated-direct-behavior value $\beta_{i}$ for $k$ route nodes is shown in (2), where $\lceil$.$\rceil is the$ nearest integer function, $w_{1}$ is the weight of $\partial p_{(r i-1, r i)}$ and correspondingly, $w_{2}$ is the weight of $l \partial_{(r i-1, r i)}$.

$$
\beta_{i}=\left\lceil\left(w_{1} \times \partial p_{(r i-1, r i)}+w_{2} \times l \partial_{(r i-1, r i)}\right)\right\rceil, \quad i \in\{1, k\} .
$$

The weights $w_{1}$ and $w_{2}$ will be $w_{1}+w_{2}=1$. The values of $w_{1}$ and $w_{2}$ are calculated as in (3), where $N(s)_{(i-1, i)} \in\{0,1\}$ is the total number of successful interactions 
between $r n_{i-1}$ and $r n_{i}$, and $\theta_{(i-1, i)} \in\{0,1\}$ is the positive feedback factor as in (Xiaoyong et al 2013).

$$
w_{1}=\frac{N(s)_{(i-1, i)}}{N(s)_{(i-1, i)}+\theta_{(i-1, i)}}, \quad w_{2}=\frac{\theta_{(i-1, i)}}{N(s)_{(i-1, i)}+\theta_{(i-1, i)}} .
$$

\subsection{Indirect behavior observation evaluation}

In Indirect Behavior Observation, each route node receives its behavior value not from the route path but from all the neighboring paths that pass through it. The routes that pass through the route node for different sources and sinks are called as the neighboring paths $n p$. The one hop neighbor node to each route node in the neighboring paths is identified as neighboring nodes $N n$. For $m$ neighboring paths $\left\{n p_{j} \mid j \in[1, m]\right\}$ that pass through a route node, $m$ neighboring nodes are identified and are denoted as $\left\{N n_{j} \mid j \in[1, m]\right\}$. Out of $m$ paths, if only $s$ paths have past transaction with $r n_{i}$, then $r n_{i}$ 's behavior is calculated using $s N n$ nodes. In this paper, $m$ neighboring paths are considered for all route nodes.

The two indirect-behavior values $I b_{v}$ are calculated by first calculating $\partial p_{(r i, N j)}$ and $l \partial_{(r i, N j)}$ values, where $\partial p_{(r i, N j)}$ and $l \partial_{(r i, N j)}$ are the probability of trust and link delay between a route node and $m$ neighbor nodes respectively. For a single route node, $m \partial p_{(r i, N j)} \mathrm{s}$ and $m l \partial_{(r i, N j)}$ $\mathrm{s}$ are generated and are represented as $\left\{\partial p_{(r i, N j)}, l \partial_{(r i, N j)} \mid i \in[1, k], j \in[1, m]\right\}$. The generated $m$ values are averaged separately to obtain indirect-probability $\lambda p_{r i}$ and indirect-link $\lambda d_{r i}$ respectively, together known as indirect-behavior values $I b_{v}$. Likewise, $I b_{v}$ is calculated for $k$ route nodes in route path as in (4). For $k r n, k I b_{v} \mathrm{~s}$ are calculated, $I b_{v}(i)=$ $\left\{\left(\lambda p_{r 1}, \lambda d_{r 1}\right), \ldots,\left(\lambda p_{r k}, \lambda d_{r k}\right)\right\}$, where $\lambda p_{r 1}$ and $\lambda d_{r 1}$ are the indirect-probability and indirectlink of the first route node.

$$
I b_{v}(i)=\left\{\left(\lambda p_{r i}=\sum_{j=1}^{m} \partial p_{(r i, N j)}\right),\left(\lambda d_{r i}=\sum_{j=1}^{m} l \partial_{(r i, N j)}\right)\right\}, \quad \forall i \in\{1, k\}, j \in\{1, m\} .
$$

The calculated two $I b_{v} \mathrm{~s}$, the first $\partial p_{(r i, N j)}$ and the second $l \partial_{(r i, N j)}$ are aggregated. The aggregated-indirect-behavior value $\eta_{i}$ is given in (5), where $\eta_{1}$ and $\eta_{k}$ are the aggregatedindirect-behavior of first $r n$ and $k^{\text {th }} r n$ and the weights of $x_{1}$ and $x_{2}$ is $x_{1}+x_{2}=1$.

$$
\eta_{i}=\left\lceil\left(x_{1} \times \lambda t_{r i}+x_{2} \times \lambda d_{r i}\right)\right\rceil, \quad i \in\{1, k\} .
$$

The $I b_{v}$ and $\eta_{i}$ values obtained by indirect observation for $k r n$ and $m N n$ is represented in table 1 with $N n$ as row and $r p$ as column. The table holds the behavior values $\left\{\left(\partial p_{(r 1, N 1)}, l \partial_{(r 1, N 1)}\right), \ldots,\left(\partial p_{(r i, N j)}, l \partial_{(r i, N j)}\right)\right\}$, the indirect-behavior values $\left\{\lambda d_{r 1}, \ldots, \lambda d_{r k}\right\}$ and the aggregated-indirect-behavior values $\left\{\eta_{1}, \ldots, \eta_{k}\right\}$ for $k \times m$ nodes with $i \in\{1, k\}$ and

\begin{tabular}{|c|c|c|c|c|c|c|c|c|c|c|}
\hline \multirow{3}{*}{$\begin{array}{l}r p \\
r n_{1}\end{array}$} & \multicolumn{7}{|c|}{$N n$} & \multirow{2}{*}{\multicolumn{2}{|c|}{$I b_{v}$}} & \multirow{3}{*}{$\begin{array}{l}\eta_{i} \\
\eta_{1}\end{array}$} \\
\hline & \multicolumn{2}{|c|}{$N n_{1}$} & \multicolumn{2}{|c|}{$\mathrm{Nn}_{2}$} & $\cdots$ & \multicolumn{2}{|c|}{$N n_{m}$} & & & \\
\hline & $\partial p_{11}$ & $l \partial_{11}$ & $\partial p_{12}$ & $l \partial_{12}$ & $\cdots$ & $\partial p_{1 m}$ & $l \partial_{1 m}$ & $\lambda t_{r 1}$ & $\lambda d_{r 1}$ & \\
\hline$r n_{2}$ & $\partial p_{21}$ & $l \partial_{21}$ & $\partial p_{22}$ & $l \partial_{22}$ & $\ldots$ & $\partial p_{2 m}$ & $l \partial_{2 m}$ & $\lambda t_{r 2}$ & $\lambda d_{r 2}$ & $\eta_{2}$ \\
\hline$r n_{k}$ & $\partial p_{k 1}$ & $l \partial_{k 1}$ & $\partial p_{k 2}$ & $l \partial_{k 2}$ & $\ldots$ & $\partial p_{k m}$ & $l \partial_{k m}$ & $\lambda t_{r k}$ & $\lambda d_{r k}$ & $\eta_{k}$ \\
\hline
\end{tabular}
$j \in\{1, m\}$.

Table 1. Behavior values using indirect observation. 


\section{Reliability factor identification methods}

In this module, three methods have been presented for identifying the reliability factor of a node using the behavior value. Among the three methods, the third method possesses better reliability.

\subsection{Indirect based reliability (IBR) method}

In IBR method, a route node identifies its reliability factor by obtaining the behavior value using indirect behavior observation and it relies on all the neighboring paths that pass through it. It is based on the heuristic that if packet loss occurs due to other causes such as poor channel conditions or normal collision by some other nodes of the neighboring paths, a low reliability value is assigned to the route node. There is also a possibility for an innocent node to be framed as bad node because the behavior value is completely decided by the neighboring paths and not by the route path. With this method, $I B R$-reliability-factor $i b r_{(i)}$ is generated using the two indirectbehavior values $\lambda p_{r i}, \lambda d_{r i}$, and their aggregated-indirect-behavior value $\eta_{i}$ as in (6). These three values form the reliability value of a route node using IBR method. The generated $i b r_{(i)}$ for $k$ route nodes $\left\{i b r_{(i)} \mid i \in[1, k]\right\}$ are recorded in the indirect set $i b-s=\left\{i b r_{(1)}, \ldots, i b r_{(k)}\right\}$, where $i b r_{(1)}$ is the $I B R$-reliability-factor for the first route node. For each route node, $i b r_{(i)}$ has three values.

$$
i b r_{(i)}=\left\{\left\langle\lambda p_{r i}\right\rangle,\left\langle\lambda d_{r i}\right\rangle,\left\langle\eta_{i}\right\rangle\right\}, \quad \forall i \in\{1, k\}
$$

\subsection{Direct based reliability $(D B R)$ method}

In DBR method, a route node identifies its reliability factor by obtaining the behavior value using direct behavior observation and it purely depends on its previous route node of the same path. When compared to IBR method, there is less possibility for an innocent node to be framed as bad node because its behavior value is decided by the route node. The $D B R$-reliability-factor $d b r_{(i)}$ is generated using two direct-behaviors values $\partial p_{(r i-1, r i)}, l \partial_{(r i-1, r i)}$ and its aggregateddirect-behavior $\beta_{i}$ represented as $d b r_{(i)}=\left\{\left(\partial p_{(r i-1, r i)}, l \partial_{(r i-1, r i)}, \beta_{i}\right) \mid i \in[1, k]\right\}$. These three values form the reliability value of a route node using DBR method. The generated $d b r_{(i)}$ for $k$ $r n,\left\{d b r_{(i)} \mid i \in[1, k]\right\}$ is recorded in the direct set $d b-s=\left\{d b r_{(1)}, \ldots, d b r_{(k)}\right\}$, where $d b r_{(1)}$ is the $D B R$-reliability-factor for the first route node.

\subsection{Hybrid based reliability (HBR) method}

Unlike prior methods, which rely on either direct observation or indirect observation, the HBR method identifies its reliability factor by obtaining the behavior value using both direct and indirect observations. When compared to IBR and DBR methods, this method has very less possibility for an innocent node to be framed as bad. In HBR method, each route node receives its behavior value from the route path by using direct observation as well as from the neighboring paths by using indirect observation. These behavior values are averaged and aggregated to obtain the aggregated-direct-behavior $\beta_{i}$ as in Section 4.2 and correspondingly, the aggregated-indirectbehavior $\eta_{i}$ as in Section 4.3, together known as hybrid-behavior $H b_{v}=\left\{\beta_{i}, \eta_{i}\right\}$. The two values of $H b_{v}$ are again aggregated to obtain aggregated-hybrid-behavior $C_{i}$ fork $r n$ as shown in (7).

$$
C_{i}=\left\lceil\left(w_{1} \times \beta_{i}+w_{2} \times \eta_{i}\right)\right\rceil, \quad i \in\{1, k\} .
$$


The $H B R$-reliability-factor $h b r_{(i)}$ is generated using $\beta_{i}, \eta_{i}$ and $C_{i}$ values. These three values form the reliability value of a route node using HBR method. The $h b r_{(i)}$ for $k r n\left\{h b r_{(i)} \mid i \in\right.$ $[1, k]\}$ is recorded in the hybrid behavior set $h b-s=\left\{h b r_{(1)}, \ldots, h b r_{(k)}\right\}$ where $h b r_{(1)}$ denotes the $H B R$-reliability-factor for the first route node.

\section{Node positioning classification (NPC)}

In this section, using the reliability value, the route nodes are level positioned and classified into groups by neuro-fuzzy classifier.

\subsection{Route node reliability levels}

The reliability factors $i b r_{(i)}, d b r_{(i)}, h b r_{(i)}$ recorded in the behavior sets $i b-s, d b-s, h b-s$ represent the reliability value of nodes using IBR, DBR and HBR methods. The reliability factor has three values and these three values form the reliability of a route node. Reliability value of a node lies between 0 and 1 . A node is identified individually based on its reliability value, if the value lies between 0 and 0.3 , then the node is identified as poor reliability level $P_{L}$, if it lies between 0.4 and 0.6, it is in moderate reliability level $M_{L}$ and from 0.7 to 1 means good reliability level $G_{L}$.

Based on these reliability levels, the ANFIS classifier categorizes the route nodes into three groups $G_{i}=\left\{G_{1}, G_{2}, G_{3}\right\}$. Group $G_{1}, G_{2}$, and $G_{3}$ corresponds to suspicious group, normal group, and trusted group respectively. A sample training node reliability level for 10 route nodes using HBR method is represented in table 2 , with route nodes as columns and reliability values of $\operatorname{HBR}\left(\beta_{i}, \eta_{i}, C_{i}\right)$ as rows. For example, if a route node's $\beta_{i}$ is in moderate reliability level, while $\eta_{i}$ and $C_{i}$ are in good levels, then ANFIS classifies the node and place it in trusted group $\mathrm{G}_{3}$.

\subsection{Interference neuro-fuzzy classifier}

ANFIS is a neuro-fuzzy system using the learning techniques of neural network (Mar et al 2010; Singh et al 2012).

Two fuzzy if-then rules based on a first order Sugeno model is considered for modeling ANFIS classifier (Tomohiro \& Michio 1985). ANFIS classifier uses five layers of nodes; with the first and the fourth layer nodes are modifiable while the rest are fixed. The parameters of modifiable

Table 2. Training node reliability level model.

\begin{tabular}{lcccc}
\hline$r n_{i}$ & $\beta_{i}$ & $\eta_{i}$ & $C_{i}$ & $G_{i}$ \\
\hline$r n_{1}$ & $P_{L}$ & $P_{L}$ & $P_{L}$ & $G_{1}$ \\
$r n_{2}$ & $M_{L}$ & $G_{L}$ & $G_{L}$ & $G_{3}$ \\
$r n_{3}$ & $P_{L}$ & $M_{L}$ & $G_{L}$ & $G_{2}$ \\
$r n_{4}$ & $P_{L}$ & $P_{L}$ & $G_{L}$ & $G_{2}$ \\
$r n_{5}$ & $G_{L}$ & $G_{L}$ & $G_{L}$ & $G_{3}$ \\
$r n_{6}$ & $M_{L}$ & $M_{L}$ & $M_{L}$ & $G_{2}$ \\
$r n_{7}$ & $P_{L}$ & $M_{L}$ & $M_{L}$ & $G_{1}$ \\
$r n_{8}$ & $P_{L}$ & $G_{L}$ & $G_{L}$ & $G_{3}$ \\
$r n_{9}$ & $P_{L}$ & $P_{L}$ & $M_{L}$ & $G_{1}$ \\
$r n_{10}$ & $M_{L}$ & $M_{L}$ & $G_{L}$ & $G_{3}$ \\
\hline
\end{tabular}


nodes are updated per iteration. The parameters $\left\{p_{i}, q_{i}, r_{i}\right\}$ and $\left\{a_{i}, b_{i}, c_{i}, d_{i}\right\}$ of layer one and four are modifiable with $\left\{x_{1}, x_{2}, x_{3}\right\}$ as three inputs and $\left\{R_{i}, S_{i}, T_{i}\right\}$ as fuzzy sets. Fuzzy rule with $i \in\{1,2\}$ is specified within the fuzzy region with $\theta_{i}$ as the output. The equivalent block diagram of Neuro-Fuzzy Interference is represented in figure 2.

The output $H(1)$ of layer one is denoted in (8). Any membership function can be used in $M\left(R_{i}\left(x_{1}\right)\right), M\left(S_{i}\left(x_{2}\right)\right)$ and $M\left(T_{i}\left(x_{3}\right)\right)$ with $\left\{p_{i}, q_{i}, r_{i}\right\}$ being the parameters of membership function as in (9).

$$
\begin{gathered}
H(1)=M\left(R_{i}\left(x_{1}\right)\right), \quad H(1)=M\left(S_{i}\left(x_{2}\right)\right), \quad H(1)=M\left(T_{i}\left(x_{3}\right)\right), \quad \forall i \in\{1,2\} \quad \text { (8) } \\
H(1)=\frac{1}{\left(1+\left(\frac{x_{1}-r_{i}}{p_{i}}\right)^{2}\right)^{q_{i}}}, H(1)=\frac{1}{\left(1+\left(\frac{x_{2}-r_{i}}{p_{i}}\right)^{2}\right)^{q_{i}}}, H(1)=\frac{1}{\left(1+\left(\frac{x_{3}-r_{i}}{p_{i}}\right)^{2}\right)^{q_{i}}}, \forall i \in\{1,2\} .
\end{gathered}
$$

In layer two, nodes are labeled as $\mathrm{U}$ and its output $H(2)$ is given in (10).

$$
H(2)=D_{i}=M\left(R_{i}\left(x_{1}\right)\right) \times M\left(S_{i}\left(x_{2}\right)\right) \times M\left(T_{i}\left(x_{3}\right)\right), \quad \forall i \in\{1,2\} .
$$

In layer three, the nodes are labeled as $\mathrm{V}$ and in layer four, nodes are labeled as $\mathrm{W}$, and their output $H(3)$ and $H(4)$ is shown in (11) and (12) respectively. The output $H(4)$ is the product of $D_{i}^{*}$ and $\theta_{i}$.

$$
H(3)=D_{i}^{*}=\frac{D_{i}}{D_{1}+D_{2}}, \quad \forall i \in\{1,2\}
$$

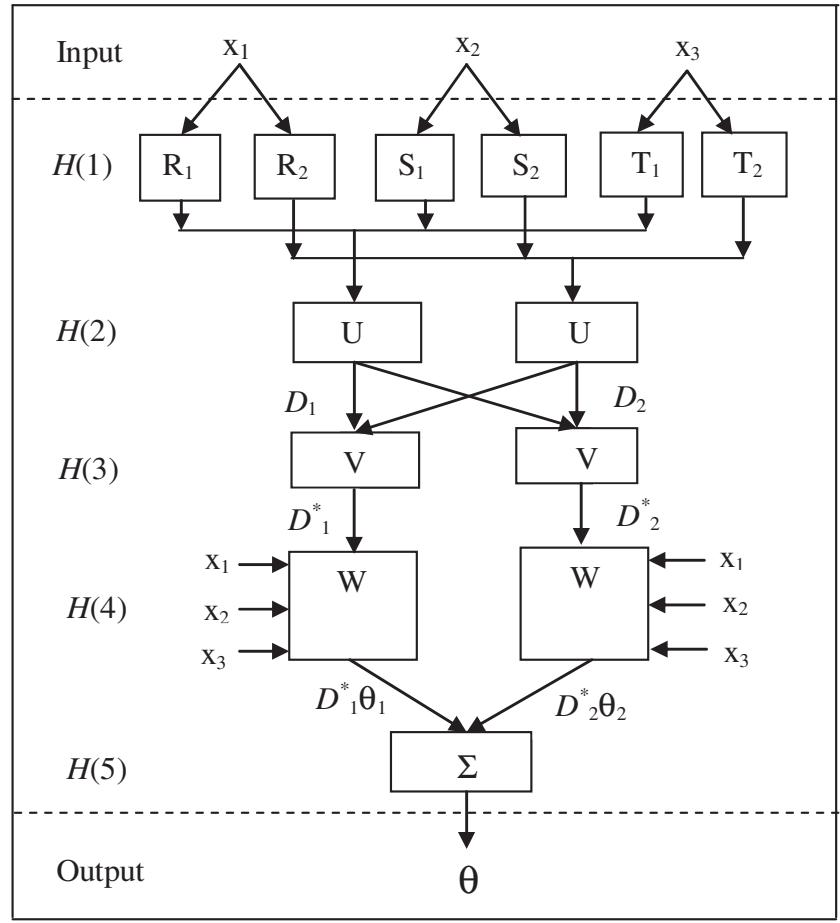

Figure 2. Neuro fuzzy interference structure. 


$$
H(4)=D_{i}^{*} \theta_{i}=D_{i}^{*}\left(a_{i} x_{1}+b_{i} x_{2}+c_{i} x_{3}+d_{i}\right), \quad \forall i \in\{1,2\} .
$$

Layer five summarizes all incoming signals on a single node and its overall output $H(5)$ is given in (13).

$$
H(5)=\sum_{i=1}^{2} D_{i}^{*} \theta_{i}, \quad \forall i \in\{1,2\} \text {. }
$$

Using the input training data, ANFIS adjusts all the parameters to make the output match the training data. ANFIS classifies the route nodes into three groups $G_{1}, G_{2}$, and $G_{3}$.

\subsection{Identifying malicious node from suspicious group}

The nodes in groups $G_{2}$ and $G_{3}$ have less probability to have jammer in it and are trusted ones, whereas nodes in group $G_{1}$ have high probability to be suspicious. Direct bit observation is obtained for all nodes in $G_{1}$. In direct bit observation, each node calculates its bit delivery ratio from its previous node in the route path. If the number of bits successfully entering the node equals to the number of bits successfully entering the next node, then the bit delivery ratio is set as one and it is a normal node. If the condition is not satisfied, it is set as zero and is put in a jammer node. The reason is that jammer node injects surplus amount of error bits into the packet. The identified jammer nodes from groups $G_{1}$ are eliminated. After elimination, the data can be securely transmitted from the source to the sink without any jamming effect. Hence jamming is prevented first by identification and then elimination.

\section{Performance evaluation}

The experimental results of Reliability Behavior Neuro-Fuzzy System are presented in this section. With the aid of ns-2, the effectiveness and efficiency of the proposed system is evaluated. Simulation is run on a $500 \times 500 \mathrm{~m}^{2}$ network with a random topology of 100 nodes. The network performance is measured by detection rate and false positive probability as well as through throughput, packet delivery ratio and delay. The ratio of the number of correctly identified jammer nodes to the total misbehaving nodes is known as detection rate, while the ratio of misidentified nodes over all malicious nodes is false positive Liu et al (2007).

\subsection{Impact of network size on increasing malicious nodes}

In the first experiment, the throughput, the packet delivery ratio and the end to end delay are demonstrated in terms of network size for each jamming strategy. Packet delivery ratio is computed by dividing the number of packets received by the number of packets sent. Figure $3 \mathrm{a}, \mathrm{b}$ and $\mathrm{c}$ shows the network performance for different number of malicious jammer nodes (MJN) under increasing network nodes. Generally, the less the number of MJN, the higher will be the throughput and packet delivery ratio, with decreasing delay. From the figure, it is observed that, as the number of MJN increases for increasing number of nodes, the throughput and packet delivery ratio decrease, while delay increases. The network performance is very sensitive to MJN.

\subsection{Evaluation of factor identification methods}

The second experiment shows the detection rate and false positive probability for the three factor identification methods under different percentage of jammers. There exist two kinds of nodes 


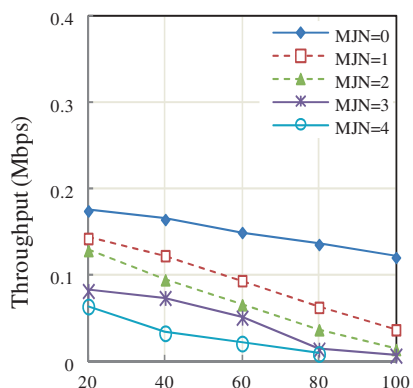

(a)

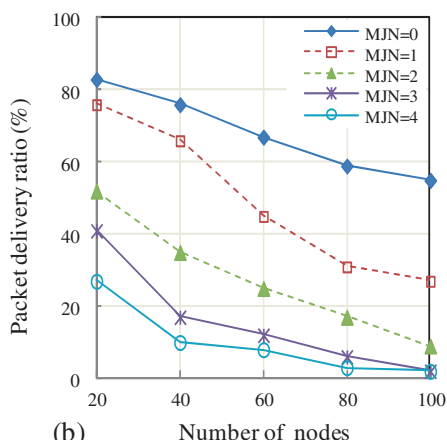

(b)

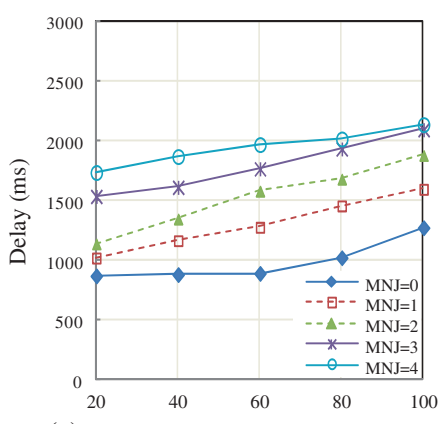

(c)

Figure 3. With increasing network size (a) Throughput, (b) Packet Delivery Ratio, and (c) End to End Delay vs. Number of nodes.

based on their activities (i) Malicious Jammer Node (MJN) and (ii) Good Normal Node (GNN). In figure $4 \mathrm{a}-\mathrm{f}$, detection rate and false positive are plotted for six MJN-GNN models, where 10$90 \%$ represents $10 \% \mathrm{MJN}$ and $90 \%$ GNN respectively. From the figure, it is observed that HBR method provides higher detection rate than the other two methods. In terms of false positive probability, IBR and DBR methods provide higher false positive probability than that of HBR. This is because the DBR and IBR methods identify malicious nodes based on direct and indirect observations respectively; therefore, there is possibility for an innocent node to be identified as

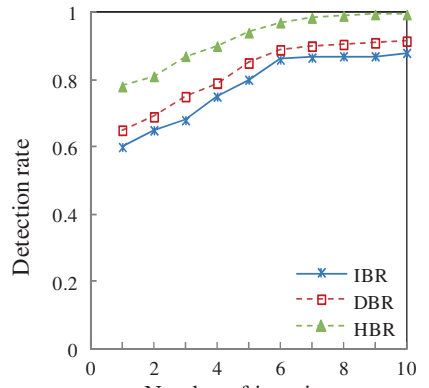

(a)

$$
10-90 \%
$$

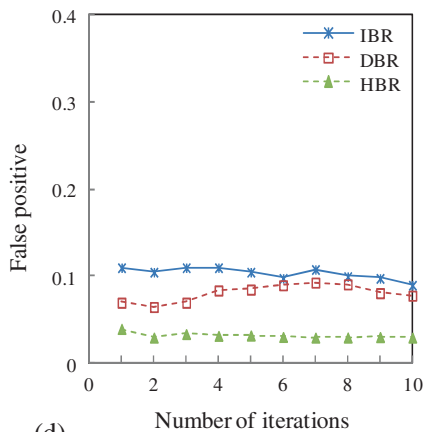

(d) $20-80 \%$

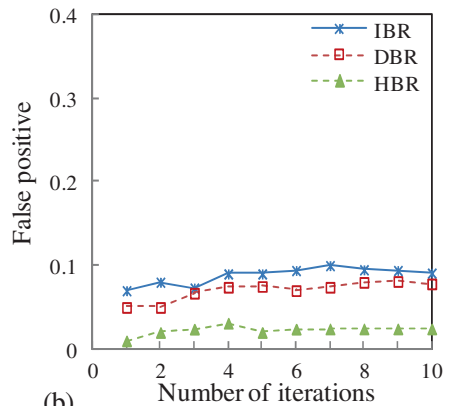

(b)

$10-90 \%$

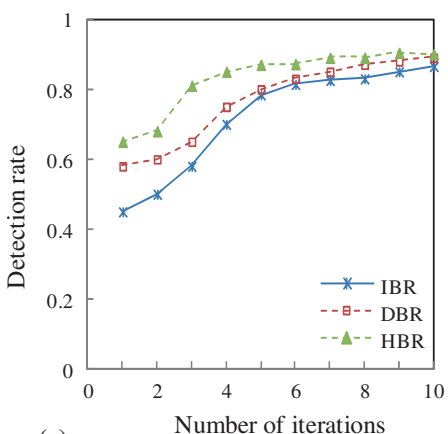

(e) $30-70 \%$

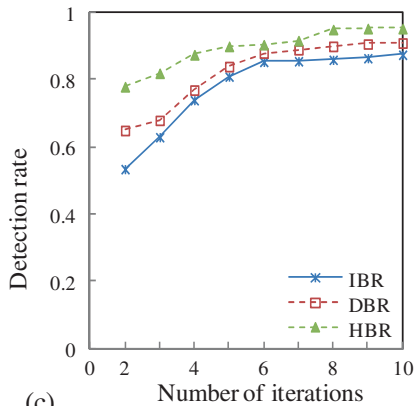

$20-80 \%$

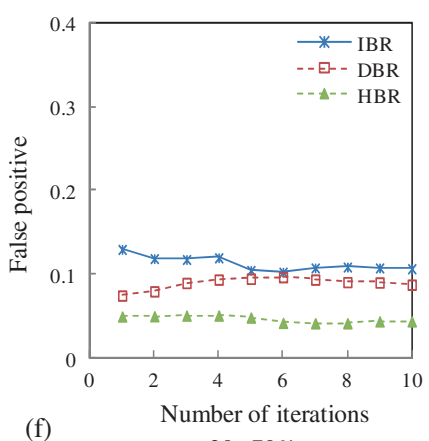

$30-70 \%$

Figure 4. Comparing MJN-GNN models for three factor identification methods. 
Table 3. Th and PDR for factor identification methods.

\begin{tabular}{|c|c|c|c|c|c|c|c|c|}
\hline \multirow[t]{2}{*}{ MJN } & \multicolumn{2}{|c|}{ No defence } & \multicolumn{2}{|c|}{ IBR } & \multicolumn{2}{|c|}{ DBR } & \multicolumn{2}{|c|}{ HBR } \\
\hline & Th & $\mathrm{Pdr}$ & Th & $\mathrm{Pdr}$ & Th & $\mathrm{Pdr}$ & Th & $\mathrm{Pdr}$ \\
\hline 2 & 0.129 & 52 & 0.134 & 65 & 0.139 & 68 & 0.16 & 77 \\
\hline 4 & 0.06 & 27 & 0.08 & 42 & 0.12 & 48 & 0.155 & 65 \\
\hline 6 & 0.03 & 19 & 0.07 & 27 & 0.1 & 31 & 0.143 & 52 \\
\hline 8 & 0.01 & 13 & 0.04 & 20 & 0.07 & 25 & 0.11 & 35 \\
\hline
\end{tabular}

suspicious. On the contrary, HBR method selects the reliability based on both direct and indirect observations, which results in less number of mis-accusations. It is also observed that as the percentage of MJN increases, detection rate decreases and false positive probability increases. Considering both the metrics, it is determined that HBR is the best reliability method among the three because of its higher detection rate and lower false positive. In HBR, the detection rate is low at the initial iterations but becomes constant after the eighth iteration.

In table 3, the throughput $\mathrm{Th}$ and the packet delivery ratio (Pdr) are demonstrated for No Defense, IBR, DBR and HBR methods. To compare the performance of different methods, the number of neighboring paths is set as 10 . The following observations can be made. First, HBR performs the best with high throughput and high packet delivery ratio. For example, when there are eight malicious nodes, the throughput of IBR is only 0.04 mbps while the PDR is $20 \%$, meaning that it does not properly identify malicious nodes. Second, DBR outperforms IBR; the reason is, DBR focuses on the feedback received from the route node.

\subsection{Evaluation on number of iterations}

In the third experiment, figure $5 \mathrm{a}$ and $\mathrm{b}$ shows the number of iterations (rounds) needed to obtain high detection rate and low false positive under four different percentage of MJN using HBR method. From the figure it is observed that, as the number of iteration increases, detection rate increases, but degrades when the percentage of MJN starts increasing. On the contrary, as the number of iteration increases, false positive decreases first and then increases as the number of jammer node increases. In figure $4 \mathrm{c}$ and $\mathrm{d}$, as the number of jammer node increases, the detection rate decreases and false positive increases for increasing iterations.

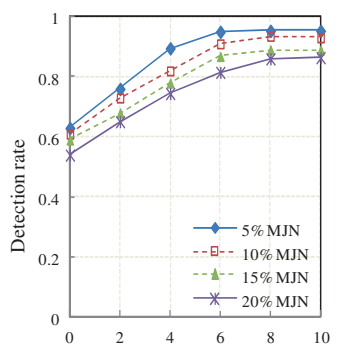

(a) Number of iterations

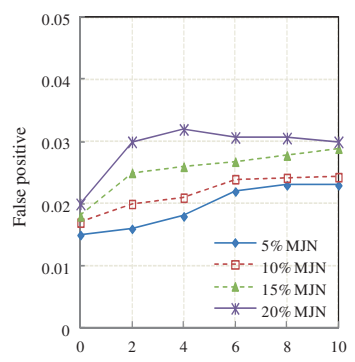

(b) Number of iterations

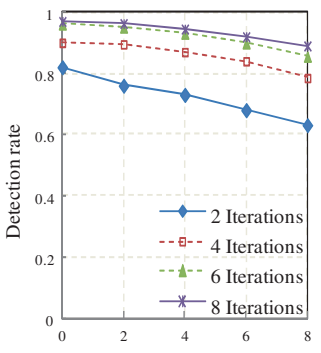

(c) Malicious jammer node

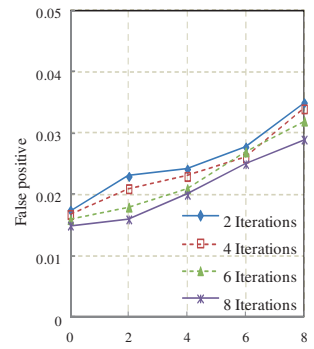

(d) Malicious jammer node

Figure 5. For different percentage of MJN (a) Detection rate and (b) False positive vs. number of iterations. With increasing iterations (c) Detection rate and (d) False positive vs. Malicious jammer node. 


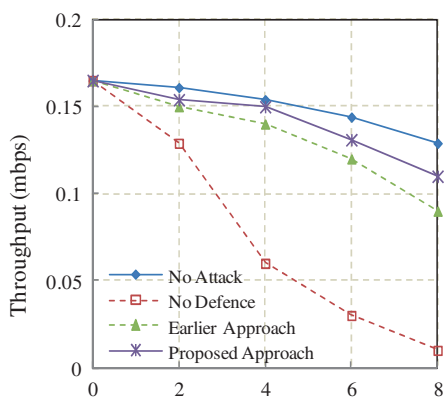

(a) Malicious jammer nodes

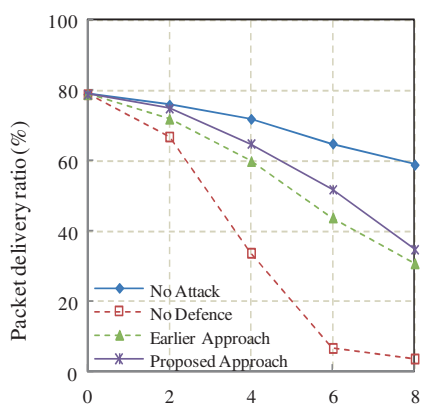

(b) Malicious jammer nodes

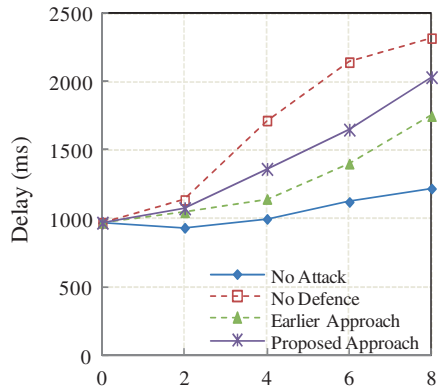

(c) Malicious jammer nodes

Figure 6. (a) Throughput, (b) packet delivery ratio, and (c) delay under: (i) no attack (ii) no defense (iii) earlier approach (iv) proposed approach vs. number of malicious nodes.

Table 4. Throughput comparison of different approaches for increasing jammers.

\begin{tabular}{|c|c|c|c|c|c|c|c|c|}
\hline \multirow[t]{3}{*}{ Approaches } & \multicolumn{8}{|c|}{ MJN } \\
\hline & \multicolumn{2}{|r|}{2} & \multicolumn{2}{|r|}{4} & \multicolumn{2}{|r|}{6} & \multicolumn{2}{|r|}{8} \\
\hline & NTH & $\mathrm{JTH} \times 10^{-2}$ & $\mathrm{NTH}$ & $\mathrm{JTH} \times 10^{-2}$ & $\mathrm{NTH}$ & $\mathrm{JTH} \times 10^{-2}$ & NTH & $\mathrm{JTH} \times 10^{-2}$ \\
\hline No attack & 0.161 & 0 & 0.154 & 0 & 0.144 & 0 & 0.129 & 0 \\
\hline Attack with no prevention & 0.129 & 0.08 & 0.06 & 0.15 & 0.03 & 0.23 & 0.01 & 0.35 \\
\hline $\begin{array}{l}\text { Prevention using researcher } \\
\text { earlier MMMTR approach }\end{array}$ & 0.15 & 0.065 & 0.14 & 0.093 & 0.12 & 0.18 & 0.09 & 0.21 \\
\hline $\begin{array}{l}\text { Prevention using Proposed } \\
\text { HBR approach }\end{array}$ & 0.154 & 0.06 & 0.15 & 0.09 & 0.131 & 0.15 & 0.11 & 0.18 \\
\hline
\end{tabular}

\subsection{Evaluation of different approaches}

In the fourth experiment, figure 6a, b and c shows the results of No Attack, No Defence, prevention using researcher's Earlier MMMTR Approach (Ratna et al 2014), and the Proposed system with HBR Approach for different number of MJN.

The proposed approach provides better network performance than the earlier work because MMMTR depends only on direct observation, whereas the proposed HBR approach depends on both direct and indirect observations.

Table 4 shows the throughput comparison of different approaches for increasing number of jammers. The network throughput (NTH) in mbps is compared with the jammer throughput $(\mathrm{JTH})$ in mbps; the amount of energy the jammer spent in corrupting the packets is measured as JTH. As JTH increases automatically the NTH decreases. Compared to the earlier work, the proposed approach is able to reduce the JTH efficiently.

\section{Conclusion}

This paper addresses the problem of physical layer jamming attack in wireless networks. In this paper, the Reliability Behavior Neuro-Fuzzy system has been proposed for preventing jamming attack based on the reliability of node. The proposed scheme first calculates the behavior value of each node in the route path using direct and indirect observations and identifies the reliability using the behavior value. Three identification methods have been presented to identify the reliability of the node. Based on the reliability value, the nodes are classified into groups using 
neuro-fuzzy classifier. Then the malicious nodes are identified and eliminated from the suspicious node group in node positioning section. Simulation result shows that HBR method yields better performance even when the jamming ratio increases and it also limits the distorting ability of the jammer. It is experimentally verified that compared to the earlier MMMTR approach, HBR provides higher detection rate of 0.99 and lower false positive probability of 0.024 at the eighth run. It is also observed that the proposed scheme attains higher network throughput and higher packet delivery ratio while attaining lower delay and lower jamming throughput.

\section{Acknowledgements}

This work was supported in part by Anna University, Chennai recognized research center lab at Francis Xavier Engineering College, Tirunelveli, India.

\section{References}

Adnane A, Bidan C and Rafael T S 2013 Trust-based security for the OLSR routing protocol. Comput. Commun.

Amariucai G T 2009 Physical layer security in wireless networks: Intelligent jamming and Eavesdropping. Ph.D. Louisiana State University. Louisiana

Bao F, Chen I R, Chang M J and Cho J H 2012 Hierarchical trust management for wireless sensor networks and its applications to trust-based routing and intrusion detection. IEEE Trans. Network Service Manag. 9(2): 169-183

Chiang J T and Hu Y C 2011 Cross-layer jamming detection and mitigation in wireless broadcast networks. IEEE/ACM Trans. Networking 19(1): 1063-6692

Furdek M and Kapov N S 2013 Attack-survivable routing and wavelength assignment for high-power jamming. In: $17^{\text {th }}$ international conference optical network design and modeling (ONDM). 70-75

Josang A, Ismail R and Boyd C 2007 A survey of trust and reputation systems for online service provision. Decis. Support Syst. 43(2): 618-644

Li C, Dai H, Xiao L and Ning P 2012 Communication efficiency of anti-jamming broadcast in large-scale multi-channel wireless networks. IEEE Trans. Signal Process. 60(10): 5281-5292

Liu H, Frias J D and Hongxun S M 2007 Using a two-timer scheme to detect selfish nodes in mobile ad-hoc networks. Proceedings of international conference on communications, internet and information technology 38(1): 40-46

Mar J, Yeh Y C and Hsiao I F 2010 An ANFIS-IDS against de-authentication DOS attacks for a WLAN. International symposium on information theory and its applications (ISITA) 548-553

Mustafa H, Zhang X, Liu Z, Xu W and Perrig A 2012 Jamming-resilient multipath routing. IEEE Trans. Dependable Secure Comput. 9(6): 852-864

Nguyen H, Pongthawornkamol T and Nahrstedt K 2011 Alibi framework for identifying reactive jamming nodes in wireless LAN. IEEE Globecom

Noubir G and Lin G 2003 Low-power DoS attacks in data wireless lans and countermeasures. Mobile Comput. Commun. Rev. 7(3): 29-30

Pelechrinis K, Koufogiannakis C and Krishnamurthy S V 2010 On the efficacy of frequency hopping in coping with jamming attacks in 802.11 networks. IEEE Trans. Wireless Commun. 9(10): 3258-3271

Proano A and Lazos L 2012 Packet-hiding methods for preventing selective jamming attacks. IEEE Trans. Dependable Secure Comput. 9(1): 101-114

Ratna S R, Ravi R and Beulah S 2014 An intelligent approach based on neuro- fuzzy detachment scheme for preventing jamming attack in wireless networks. J. Intell. Fuzzy Logic. doi: 10.3233/IFS-141363. ISSN 1064-1246(Print). 1875-8967 Online (in press)

Richa A, Scheideler C, Schmid S and Zhang J 2013 An efficient and fair MAC protocol robust to reactive interference. IEEE/ACM Trans. Networking 21(3): 760-771 
Sarker J H and Mouftah H T 2012 Mitigating the effect of jamming signals in wireless ad hoc and sensor networks. IET Commun. 6(3): 311-317

Shila D M, Yu C and Anjali T 2010 Mitigating selective forwarding attacks with a channel-aware approach in WMNs. IEEE Trans. Wireless Commun. 9(5): 1661-1675

Shiu Y S, Chang S Y, Wu H C, Huang S C H and Chen H H 2011 Physical layer security in wireless networks: A tutorial. IEEE Wireless Commun. 18(2): 66-74

Singh S, Sarje A K and Misra M 2012 Client-side counter phishing application using adaptive neuro-fuzzy inference system. Fourth international conference on computational intelligence and communication networks (CICN) 788-792

Spuhler M, Giustiniano D, Lenders V, Wilhelm M and Jens B S 2014 Detection of reactive jamming in DSSS-based wireless communications. IEEE Trans. Wireless Commun. 13(3): 1593-1603

Tomohiro T and Michio S 1985 Fuzzy identification of systems and its applications to modelling and control. IEEE Trans. Syst., Man Cybern. 15(1): 116-132

Xiaoyong L, Zhou F and Du J 2013 LDTS: A lightweight and dependable trust system for clustered wireless sensor networks. IEEE Trans. Inform. Forensics Security 8(6): 924-935

Xuan Y, Shen Y, Nguyen N P and Thai M T 2012 A trigger identification service for defending reactive jammers in WSN. IEEE Trans. Mobile Comput. 11(5): 793-806

Zhan G, Shi W and Deng J 2012 TARF: Design and implementation of TARF: A trust-aware routing framework for WSNs. IEEE Trans. Dependable Secure Comput. 9(2): 184-197 